\title{
Optical polarizabilities of large molecules measured in near-field interferometry
}

\author{
Lucia Hackermüller ${ }^{\star}{ }^{\star}$, Klaus Hornberger ${ }^{2}$, Stefan Gerlich ${ }^{1}$, Michael Gring ${ }^{1}$, Hendrik Ulbricht ${ }^{1}$, and \\ Markus Arndt ${ }^{1}$ \\ 1 Faculty of Physics, University of Vienna, Boltzmanngasse 5, A-1090 Vienna, Austria \\ 2 Arnold Sommerfeld Center for Theoretical Physics, Ludwig-Maximilians-Universität, Theresienstr. 37, D-80333 Munich, \\ Germany
}

Sent: October 26, 2018, Received: date / Revised version: date

\begin{abstract}
We discuss a novel application of matter wave interferometry to characterize the scalar optical polarizability of molecules at $532 \mathrm{~nm}$. The interferometer presented here consists of two material absorptive gratings and one central optical phase grating. The interaction of the molecules with the standing light wave ' is determined by the optical dipole force and is therefore directly dependent on the molecular polarizability at the wavelength of the diffracting laser light. By comparing the observed matter-wave interference contrast with a theoretical model for several intensities of the standing light wave and molecular velocities we can infer the polarizability in this first proof-of-principle experiment for the fullerenes $\mathrm{C}_{60}$ and $\mathrm{C}_{70}$ and we find a good agreement ' with literature values.
\end{abstract}

\section{Introduction}

Matter-wave experiments with neutrons, electrons and atoms are well-established tools for the investigation of fundamental physical concepts $1,2,3,4,4$, as well as for innovative and practical measurement applications [5], 6, 7,8. Coherence experiments with complex molecules are still relatively young [9]. But also here interesting applications have been identified, such as for instance in measurements of molecular static polarizabilities [10, 11.

Static polarizabilities and permanent dipole moments can be predicted by ab-initio and semi-classical methods [11,12] and they can be determined in molecular beam deflection experiments [13, 14. All these methods may reach an accuracy of better than ten percent but they

Send offprint requests to: markus.arndt@univie.ac.at

* present address: Johannes-Gutenberg-Universität Mainz, Institut für Physik, Staudingerweg 7, D-55120 Mainz, Germany are also increasingly demanding with increasing particle mass and complexity.

Atomic Mach-Zehnder interferometry has achieved a precision of better than $1 \%$ for lithium [15] and better than $0.1 \%$ for sodium [16], but this far-field arrangement is rather demanding in itself and it is strongly impeded for large molecules because it requires a collimation and a brilliance beyond those of existing molecular beams.

On the other hand, it has been shown, that nearfield interferometry is well-suited for less intense sources of large objects 17 and Talbot-Lau deflectometry was recently employed to measure the static polarizability of fullerenes [10] as well as of porphyrins and porphyrin derivatives [1].

All earlier interferometric polarizability experiments relied on the application of high static electric fields to shift the atomic [16,15] or molecular [10] matter wave phase in proportion to the particle's static polarizability.

Measurements of optical (AC) polarizabilities of clusters larger than diatomic molecules have not been available until a few years ago [18. As of today only very few methods have been explored and exclusively demonstrated with fullerenes: Ballard et al. were the first to exploit the recoil imparted on a $\mathrm{C}_{60}$ beam when it crossed an intense standing light wave at $1064 \mathrm{~nm} \mathrm{[18}$. Their detector was a position sensitive time-of-flight mass spectrometer which allowed to retrieve the polarizability at $1064 \mathrm{~nm}$ from a classical beam broadening.

In our present work we exploit near-field quantum diffraction in a Kapitza-Dirac-Talbot-Lau configuration [20] for retrieving the optical polarizability of both $\mathrm{C}_{70}$ and $\mathrm{C}_{60}$ from the evolution of the quantum fringe visibility as a function of the diffracting laser power. This scheme offers a higher throughput than in far-field diffraction experiments. Even in the pure quantum regime it is in principal scalable to masses beyond $10,000 \mathrm{amu}$ and it can thus yield interesting information complementary to optical spectroscopy experiments. 


\section{Setup}

Our new interferometer differs from an established earlier version [21 in that it consists of two material gratings and one central optical phase grating [20]. The first grating $\mathrm{G}_{1}$ prepares the necessary coherence of the only weakly collimated molecular beam. At the second grating $\mathrm{G}_{2}$, which is realized by a standing laser light wave at $532 \mathrm{~nm}$, diffraction is based on the optical dipole potential

$$
U(x, z)=-\frac{1}{2} \alpha_{L} E^{2}(x, z, t),
$$

with the scalar optical polarizability $\alpha_{L}$ and the electric field of the focused laser light wave $E$. Near-field matterwave interference, according to the Talbot effect, leads to self-imaging [22,23,24] of the complex transmission function of $\mathrm{G}_{2}$ into a periodic molecular density distribution at the position of the third grating $\mathrm{G}_{3}$. The latter acts as a detector screen: shifting this mask across the periodic interference pattern and detecting the transmitted molecular flux then allows to reveal the molecular interference pattern.

We designate our new setup as a Kapitza-DiracTalbot-Lau (KDTL) interferometer 20, as it combines the virtue of diffraction at standing light waves [25, 26, 27,19] with the Talbot-Lau concept. The central optical grating becomes very important and useful for massive and highly polarizable particles as well as for small grating openings: For material gratings the influence of the van der Waals potential at the second grating would increase with $\alpha$ and with shrinking distance between the molecules and the grating walls. In [28,29] it has been shown that the particle-wall interaction adds a velocity and position dependent phase on the molecular wave function. In interferometry with material grat-

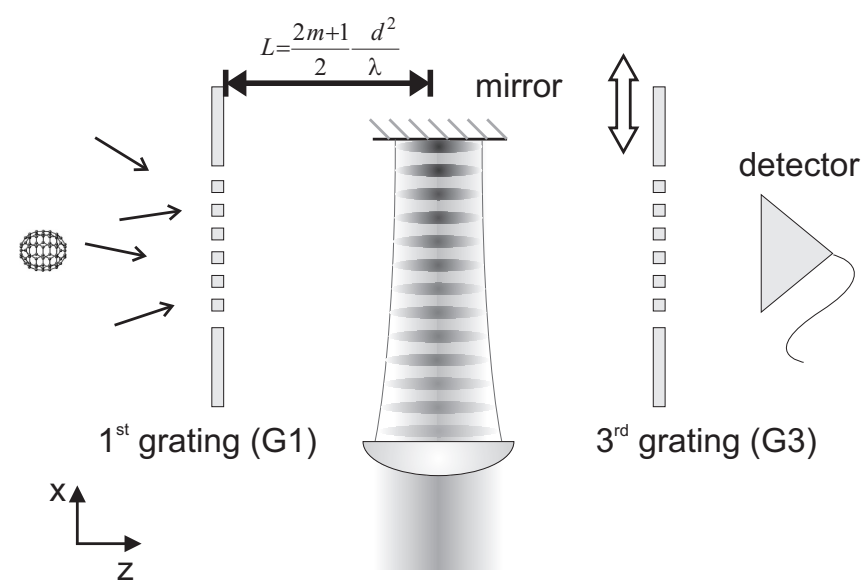

Fig. 1 Setup of the Kapitza-Dirac-Talbot-Lau interferometer: it consists of two material nanostructures and and one standing light wave. For detection, the third grating is shifted in $20 \mathrm{~nm}$ steps over the molecular pattern. The transmitted molecules are ionized and counted in an electron multiplier. ings this imposes a rather demanding experimental constraint, since high fringe contrasts can only be obtained in very narrow velocity bands, typically of the order of $\Delta v / v \leq 1 \%$ [21,20]. This requirement can however be enormously alleviated by employing optical instead of material gratings. It turns out to be sufficient to have an optical diffraction grating in the center of the KDTLinterferometer alone: The first and third material gratings serve as absorptive masks for the preparation of coherence $\left(\mathrm{G}_{1}\right)$ and the spatially resolved and parallel detection $\left(\mathrm{G}_{3}\right)$ of the wide molecular beam. Additional phases which are imprinted onto the molecules by these structures, play no significant role for the formation of the interference pattern.

The $\mathrm{SiN}_{x}$ material gratings have a period of $\mathrm{d}=266 \mathrm{~nm}$, a thickness of $190 \mathrm{~nm}$ and an open fraction of $\mathrm{f}=0.42$. The latter is the ratio between slit opening and grating period. The nanomasks were manufactured by T. Savas (MIT, Cambridge, USA) with a period homogeneity of better than $\Delta \mathrm{d} \leq 1 \AA$ across the entire width of the molecular beam. In the KDTLI setup $\mathrm{G}_{1}$ and $\mathrm{G}_{3}$ are separated by a distance of $210 \mathrm{~mm}$ as sketched in Fig. 1. The center of the standing light-wave is positioned with an uncertainty of less than $20 \mu \mathrm{m}$ in the middle between these two masks. The light is derived from a green singlemode laser (Coherent Verdi $10 \mathrm{~W}$ at $532 \mathrm{~nm}$ ), tightly focused by a cylindrical lens and back reflected from a planar mirror inside the vacuum chamber.

The fullerene beam is generated by an effusive source and passes the standing light wave in a distance of less than $300 \mu \mathrm{m}$ from the mirror surface, where the laser beam is already focused to $w_{x}=20 \mu \mathrm{m}$ and $w_{y}=$ $900 \mu \mathrm{m}$. The molecules are collimated by two slits to limit the angle of incidence onto the standing light-wave to $90 \pm 0.02$ degrees. This ensures that no molecule will average nodes and an anti-nodes of the light grating.

The fullerenes are detected behind the interferometer by either laser ionization, for $\mathrm{C}_{70}$, or electron impact ionization quadrupole mass spectrometry for $\mathrm{C}_{60}$. The spatial resolution of the detector is provided by the third grating which is mounted on a piezo-electric stage. It can be shifted with a repeatability of $\sim 10 \mathrm{~nm}$. The material gratings are mounted on rotation stages in order to allow alignment around the vertical axis and around the molecular beam axis with better than $300 \mu \mathrm{rad}$ precision. The velocity of the molecules is controlled by a gravitational velocity selection scheme [21.

Interference in the Kapitza-Dirac-Talbot-Lau interferometer occurs at de Broglie wavelengths which fulfill the KDTL-condition

$$
L_{K D T L}=\frac{(2 m+1)}{2} \frac{d^{2}}{\lambda_{d B}}
$$

where $\mathrm{m}$ is an integer number. This condition, which is valid for weak laser intensities $\left(\Phi_{\max }<3.05\right.$, s. below) reveals that the interference maximum recurs with integer multiples of the Talbot period: $L_{T}=d^{2} / \lambda_{d B}$, 
but shifted by half a period in comparison to the earlier Talbot-Lau interferometer [21. For fullerenes at velocities between $100 \mathrm{~m} / \mathrm{s}$ and $190 \mathrm{~m} / \mathrm{s}$ our KDTLI-setup is operating between the fourth and the eighth Talbot diffraction order.

\section{Theoretical model}

The conservative interaction of the molecule with the electric field of the standing light-wave is described by the dipole potential of Eq. 1. The molecules thus acquire a position-dependent phase which enters the transmission function $t_{2}(x)$ of $\mathrm{G}_{2}$ :

$$
\begin{aligned}
t_{2}(x) & =\exp \left(-\frac{i}{\hbar} \int U(x, z(t), t) d t\right) \\
& =\exp \left(i \Phi_{\max } \sin ^{2}\left(\frac{2 \pi}{\lambda_{L}} x\right)\right),
\end{aligned}
$$

with a maximal phase shift at the anti-nodes of

$$
\Phi_{\max }=\frac{8 \sqrt{2 \pi} \alpha_{L}}{\hbar c w_{y} v_{z}} P
$$

where $\alpha_{L}$ is the real part of the optical polarizability at the laser wavelength $\lambda_{L}$ and $P$ the light power [19]. We further include the possibility of photon absorption, which can lead to additional transverse momentum kicks imparted onto the molecules. The absorption process is governed by Poissonian statistics and the mean number of absorbed photons in an anti-node of the standing wave is

$$
n_{0}=\frac{\sigma_{a b s}}{\hbar \omega_{L}} \int I(x, z(t), t) d t=\frac{8 \sigma_{a b s} \lambda_{L}}{\sqrt{2 \pi} h c w_{y} v_{z}} P .
$$

Here, $\sigma_{a b s}$ is the absorption cross section at the laser wavelength, determined by the imaginary part of the optical polarizability at the laser frequency $\omega_{L}$ and $I$ is the light intensity. The expected interference fringe contrast at fixed molecule velocity is then given by 20 .

$$
\begin{aligned}
V= & 2\left(\frac{\sin (\pi f)}{\pi f}\right)^{2} \exp \left(-\xi_{a b s}\right) \frac{\xi_{c o h}-\xi_{a b s}}{\xi_{c o h}+\xi_{a b s}} \\
& J_{2}\left(-\operatorname{sgn}\left(\xi_{c o h}+\xi_{a b s}\right) \sqrt{\xi_{c o h}^{2}-\xi_{a b s}^{2}}\right)
\end{aligned}
$$

where

$$
\xi_{c o h}=\Phi_{\max } \sin \left(\pi \frac{L}{L_{T}}\right),
$$

accounts for the coherent interaction and

$$
\xi_{a b s}=n_{0} \sin ^{2}\left(\frac{\pi L}{2 L_{T}}\right),
$$

\begin{tabular}{|c|c|c|c|c|}
\hline & thin films 32 & EELS 33. & theory 35 & this work \\
\hline $\mathrm{C}_{60}$ & $90 \AA^{3}$ & $98.2 \AA^{3}$ & 80.6 & $90(11) \AA^{3}$ \\
\hline $\mathrm{C}_{70}$ & $118.4 \AA^{3}$ & $122.6 \AA^{3}$ & - & $117(14) \AA^{3}$ \\
\hline
\end{tabular}

for the incoherent scattering of photons. Here $L$ is the distance between the gratings and $J_{2}$ is the Bessel function of the second kind.
Table 1 Optical polarizability of the fullerenes at $532 \mathrm{~nm}$ : The dielectric function of the thin film measurements is converted to a polarizability using a lattice constant of $a=$ $14.17 \AA$ for $\mathrm{C}_{60}$ and $a=15.2 \AA$ for $\mathrm{C}_{70} 33$.

\section{Polarizability measurements}

As can be seen from the equations above, the molecular optical scalar polarizability $\alpha_{L}$ enters directly the interference visibility of the KDTL-interferometer, through the maximum phase shift $\Phi_{\max }$. We can therefore exploit the KDTLI to determine $\alpha_{L}$ when the resonant absorption cross section $\sigma_{a b s}$ is known from separate experiments, such as gas-phase spectroscopy. In the following we compare the experimentally observed dependence of the interference contrast on the varying laser power and on the molecular velocity with the theoretical model from section 3 .

The interference patterns of $\mathrm{C}_{70}$ are recorded for eight different velocity distributions and for up to twenty different laser powers $P$. For each sinusoidal interference curve we extract the experimental fringe visibility $V=\left(S_{\max }-S_{\min }\right) /\left(S_{\max }+S_{\min }\right)$ and plot it versus the diffracting laser power, as shown in Fig. 2] We then fit the resulting curves with equation (6), using the polarizability as a single free parameter, common for all laser powers and velocities. Given the value for the optical absorption cross section [30,31 $\sigma_{a b s}(532 \mathrm{~nm})=$ $2.1 \times 10^{-17} \mathrm{~cm}^{-2}$ and including the experimentally determined velocity distributions in our theoretical model we then determine the scalar optical polarizability to be $\alpha_{L}(532 \mathrm{~nm})=117 \pm 14 \AA^{3}$.

This value is consistent with earlier results obtained from the dielectric response of thin fullerene films 32 , 33. It is also in good agreement with measurements of the gas-phase static polarizability which has been determined to be $\alpha=102 \pm 14 \AA^{3}$ using Stark deflectometry 34 and $\alpha=108.5 \pm 2.0 \pm 6.2 \AA^{3}$ by Talbot-Lau deflectometry [10]. The optical polarizability is expected to be higher than the static value since the incident light approaches (still from far away) allowed optical transitions at $532 \mathrm{~nm}$. A comparison of our work with literature values for fullerene polarizabilities at $532 \mathrm{~nm}$ is shown in Table 1

The error bars in our experiment have various different contributions: Firstly, the absolute calibration of our present optical power meter is good to within $10 \%$. This is a systematic uncertainty in the power measurement, but the reproducibility and linearity in the reading are better than $5 \%$. Secondly, the vertical waist $w_{y}$ of the laser beam enters linearly in the available intensity. In our present measurements it could been measured with 

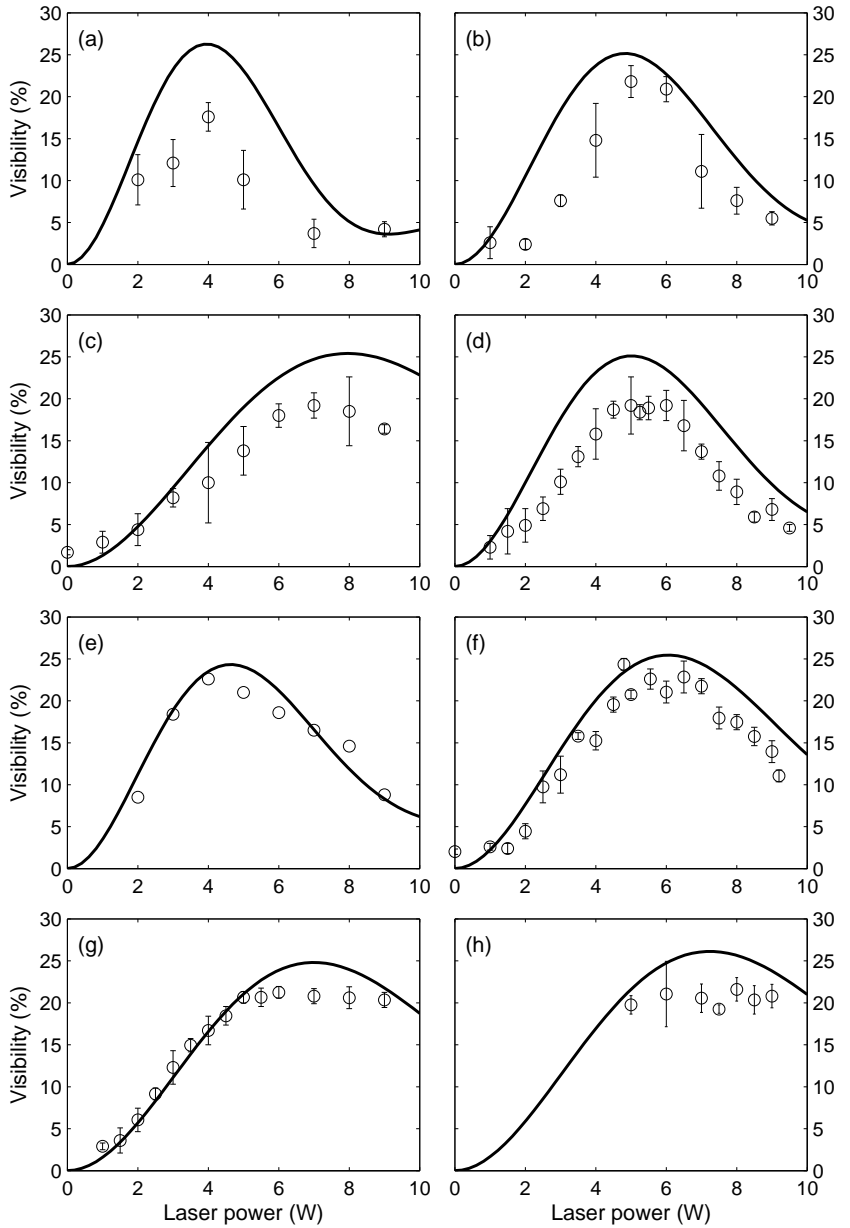

Fig. 2 Variation of the $\mathrm{C}_{70}$ interference contrast with the diffracting laser power. The velocity distributions in these experiments were characterized by the following most probable velocities $v_{m}$ and velocity spreads $\Delta v$ (standard deviation): a) $v_{m}=99.7 \mathrm{~m} / \mathrm{s}, \Delta v=18.3 \mathrm{~m} / \mathrm{s}$, b) $v_{m}=117.3 \mathrm{~m} / \mathrm{s}$, $\Delta v=14.4 \mathrm{~m} / \mathrm{s}, \quad$ c) $v_{m}=196.7 \mathrm{~m} / \mathrm{s}, \quad \Delta v=39.5 \mathrm{~m} / \mathrm{s}, \quad$ d) $v_{m}=$ $124.6 \mathrm{~m} / \mathrm{s}, \Delta v=22.8 \mathrm{~m} / \mathrm{s}$, e) $v_{m}=114.4 \mathrm{~m} / \mathrm{s}, \Delta v=18.8 \mathrm{~m} / \mathrm{s}$, f) $v_{m}=152.7 \mathrm{~m} / \mathrm{s}, \Delta v=24.8 \mathrm{~m} / \mathrm{s}$, g) $v_{m}=171.2 \mathrm{~m} / \mathrm{s}, \Delta v=$ $28.8 \mathrm{~m} / \mathrm{s}$, h) $v_{m}=179.9 \mathrm{~m} / \mathrm{s}, \Delta v=33.5 \mathrm{~m} / \mathrm{s}$. The experimental data are fitted with a single common parameter $\alpha_{L}(532 \mathrm{~nm})$ using equation (6). The error bars on the data are standard deviations taken from three consecutive recordings with the same settings. Figure e) has no error bar since only one data set was taken.

an accuracy of $5 \%$. In contrast to that, the horizontal laser waist $(20 \mu \mathrm{m})$ can only be measured with an estimated accuracy of $20 \%$, but its uncertainty cancels out in first order, since a larger waist is associated with a lower intensity but also with a longer transition time for the molecules. Thirdly, the knowledge of the optical absorption cross section is relevant for the numerical fit of the data. However it enters very weakly: even an uncertainty of $50 \%$ in $\sigma_{a b s}$ results only in a $3 \%$ variation of the fit value for the polarizability. Fourthly, there is a statistical error, as can be seen from the scatter of the data points. Taking a 'worst case scenario' by separately fit-

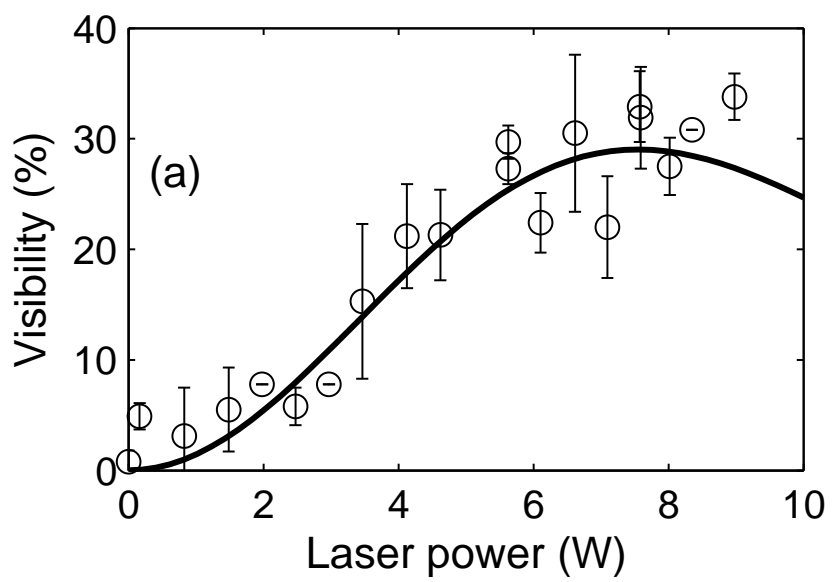

Fig. 3 Variation of the $\mathrm{C}_{60}$ interference contrast with the power of the diffracting laser light-wave. The experimental points are modeled with equation (6) and $\alpha_{L}$ as a free parameter after choosing $\sigma_{a b s}$ from the literature. The error bars correspond to the standard deviation of three consecutive measurements.

ting the upper and the lower envelope of all error bars in the data distribution, we see a maximum uncertainty of $2.3 \AA^{3}$. Systematic deviations of the interferogram from an ideal fringe contrast, which can be caused by day-today variations of the laboratory noise background, enter very weakly into the fit value. All experimental uncertainties mentioned above contribute independently and sum up to a total uncertainty of $12 \%$.

Most parameters can clearly be improved in future experiments: With an improved detection efficiency the width of the velocity distribution can probably still be reduced by an order of magnitude. The measurement of the laser power and waist may still be improved by a factor of three using commercially available sensors.

In Fig. 3 we show the power dependence of the fringe contrast of $C_{60}$ for a mean molecular velocity of $153 \mathrm{~m} / \mathrm{s}$ and a standard deviation of $\Delta v / v=0.3$. The larger error bars here are caused by the lower detection efficiency of $C_{60}$ in comparison to $C_{70}$. We again use equation 6 to fit the experimental data points and evaluate, for a given absorption cross section of $\sigma_{a b s}=3.2 \times 10^{-18} \mathrm{~cm}^{2}$ 30.36, the dynamic polarizability of $\mathrm{C}_{60}$ at $532 \mathrm{~nm}$ to be $\alpha_{L}=91(11) \AA^{3}$. This value is in good agreement with previous measurements, as shown in Table 1 and also consistent with the static polarizability values which were determined by Stark-deflectometry $\left(\alpha=76.5 \pm 8.0 \AA^{3}[14)\right.$ and by interferometric TalbotLau deflectometry $\left(\alpha=88.9 \pm 0.09 \pm 5.1 \AA^{3}[10]\right)$.

In conclusion we have used a Kapitza-Dirac-TalbotLau interferometer to determine the scalar optical polarizability of both Fullerenes $\mathrm{C}_{60}$ and $\mathrm{C}_{70}$. Our method can be extended to all volatile molecules that may be subjected to KDTL-interferometry. In the present setup this can cover a wide class of effusive beams in principle with molecular masses up to 10,000 amu. 
Combined with an independent evaluation of optical absorption cross sections the new technique can be an interesting complement to existing methods [18, 19, 13. and it will provide new data for a large set of molecules as an experimental benchmark for new computational molecular models.

\section{Acknowledgements}

This work has been supported by the Austrian Science Foundation (FWF), within the SFB projects F1505 and F1512 and START Y177. K.H. acknowledges support within the Emmy Noether program by the German Science Foundation DFG.

\section{References}

1. C. G. Shull, Phys. Rev. 179, 752 (1969).

2. G. Moellenstedt and C. Z. Joensson, Z. Phys. 155, 472 (1959).

3. O. Carnal and J. Mlynek, Phys. Rev. Lett. 66, 2689 (1991).

4. D. W. Keith, C. R. Ekstrom, Q. A. Turchette, and D. E. Pritchard, Phys. Rev. Lett. 66, 2693 (1991).

5. H. Rauch and A. Werner, Neutron Interferometry: Lessons in Experimental Quantum Mechanics (Oxford Univ. Press, Oxford 2000).

6. D. L. Price, Neutron Scattering, Part B, 23 Experimental Methods in the Physical Sciences (Academic Press Inc., New York 1996).

7. A. Tonomura, Reviews of Modern Physics 59, 639 (1987).

8. A. D. Cronin, J. Schmiedmayer, and D. E. Pritchard, Rev. Mod. Phys. (2007).

9. M. Arndt, O. Nairz, J. Voss-Andreae, C. Keller, G. V. der Zouw, and A. Zeilinger, Nature 401, 680 (1999).

10. M. Berninger, A. Stéfanov, S. Deachapunya, and M. Arndt, Phys. Rev. A (2007).

11. S. Deachapunya, P. J. Fagan, A. G. Major, E. Reiger, H. Ritsch, A. Stefanov, H. Ulbricht, and M. Arndt, http://arxiv.org/abs/0708.1449 (2007).

12. M. Frisch, G. Trucks, H. Schlegel, G. Scuseria, M. Robb, J. Cheeseman, J. Montgomery, Jr., T. Vreven, K. Kudin, et al., Gaussian 03W, Version 6.0 (Gaussian Inc., Pittsburgh, PA, 2003).

13. K. Bonin and V. Kresin, Electric-Dipole Polarizabilities of Atoms, Molecules and Clusters (World Scientific, Singapore 1997), ISBN 981-02-2493-1.

14. R. Antoine, P. Dugourd, D. Rayane, E. Benichou, M. Broyer, F. Chandezon, and C. Guet, J. Chem. Phys. 110, 9771 (1999).

15. J. M. Amini and H. Gould, Phys. Rev. Lett. 91, 153001 (2003).

16. C. Ekstrom, J. Schmiedmayer, M. Chapman, T. Hammond, and D. Pritchard, Phys. Rev. A 51, 3883 (1995).

17. J. Clauser, in Experimental Metaphysics, edited by R. Cohen, M. Horne, and J. Stachel (Kluwer Academic, Dordrecht 1997).

18. A. Ballard, K. Bonin, and J. Louderback, J. Chem. Phys. 114, 5732 (2000).
19. O. Nairz, B. Brezger, M. Arndt, and A. Zeilinger, Phys. Rev. Lett. 87, 160401 (2001).

20. S. Gerlich, L. Hackermüller, K. Hornberger, A. Stibor, H. Ulbricht, M. Gring, F. Goldfarb, T. Savas, M. Müri, M. Mayor, and M. Arndt, Nature Physics, doi:10.1038/nphys701 (2007).

21. B. Brezger, L. Hackermüller, S. Uttenthaler, J. Petschinka, M. Arndt, and A. Zeilinger, Phys. Rev. Lett. 88, 100404 (2002).

22. J. F. Clauser and S. Li, Phys. Rev. A 49, R2213 (1994).

23. B. Dubetsky and P. R. Berman, in Atom Interferometry, edited by P. R. Berman (Academic Press, San Diego 1997).

24. K. Hornberger, J. E. Sipe, and M. Arndt, Phys. Rev. A 70, 53608 (2004).

25. P. J. Martin, B. G. Oldaker, A. H. Miklich, and D. E. Pritchard, Phys. Rev. Lett. 60, 515 (1988).

26. P. L. Kapitza and P. A. M. Dirac, Proc. Camb. Philos. Soc. 29, 297 (1933).

27. D. L. Freimund, K. Aflatooni, and H. Batelaan, Nature 413, 142 (2001).

Ph.D. thesis, University of Nebraska (2003).

28. R. E. Grisenti, W. Schöllkopf, J. P. Toennies, G. C. Hegerfeldt, and T. Köhler, Phys. Rev. Lett. 83, 1755 (1999).

29. R. Brühl, P. Fouquet, R. E. Grisenti, J. P. Toennies, G. C. Hegerfeldt, T. Köhler, M. Stoll, and C. Walter, Europhys. Lett. 59, 357 (2002).

30. P. F. Coheur, M. Carleer, and R. Colin, J. Phys. B: At. Mol. Opt. Phys. 29, 4987 (1996).

31. K. Hornberger, L. Hackermüller, and M. Arndt, Physical Review A 71, 023601 (2005).

32. P. Eklund, A. Rao, Y. Wang, P. Zhou, K. Wang, J. Holden, M. Dresselhaus, and G. Dresselhaus, Thin Solid films 257, 211 (1995).

33. E. Sohmen, J. Fink, and W. Krätschmer, Z. Phys. B 86, 87 (1992).

34. I. Compagnon, R. Antoine, M. Broyer, P. Dugourd, J. Lerme, and D. Rayane, Phys. Rev. A 64, 025201 (2001).

35. K. Ruud, D. Jonsson, and P. R. Taylor, J. Chem. Phys. 114, 4331 (2001).

36. C. Ferrante, R. Signorini, A. Feis, and R. Bozio, Photochem. Photobiol. Sci. 2, 801 (2003). 